\title{
Existence et objet de la « sociologie de la littérature », aujourd'hui
}

\section{Jacques Leenhardt*}

\section{Resumé}

Pour des raisons méthodologiques, la sociologie entretient une relation plutôt distante avec la littérature. En tant que discipline scientifique, la sociologie doit définir ses objets sur la base de «propriétés inhérentes » (Durkheim). Y aurait-il une littérarité (Literarnost), telle que proposée par Jakobson ? Évidemment pas. La même chose s'applique à la fictionalité. La sociologie, par conséquent, préfère aborder son objet de façon détournée, pour aborder la « littérature » par son environnement : auditoires, critiques, politiques éditoriales, lecture. Cet article analyse quelques raisons historiques et épistémologiques d'une telle stratégie, qui évite d'aborder le noyau même de la littérature : la confrontation de différents mondes fictifs dans le texte et dans la lecture. Selon la théorie de la fiction, si la sociologie doit comprendre les forces qui transforment le statut actuel de la société, elle devrait accorder plus d'attention aux processus symboliques qui se produisent dans l'expérience littéraire, une activité qui permet à tous de se confronter des situations et des valeurs possibles (fictifs) et, par conséquent, symbolise un monde social différent possible.

Mots-clés : Littérature, Lecture de fiction, Mondes possibles, Sociologie

*École des Hautes Études en Sciences Sociales. Paris, France 


\section{Existence and object of the "sociology of literature", today}

\section{Abstract}

For methodological reasons, sociology keeps a rather distant relationship to literature. As a scientific discipline, sociology has to define its objects on the basis of "inherent properties" (Durkheim). Would there be a literariness (Literarnost), as proposed by Roman Jakobson, in 1919? Obviously, not. The same applies to fictionality. Sociology, therefore, prefers to tackle its object in a roundabout way, to approach "literature" by its surroundings: audiences, critiques, publishing policies, reading. This paper analyzes some historical and epistemological reasons for such a strategy, which avoids addressing the very core of literature: the confrontation of different fictional worlds in the text and in the reading. Based on the fiction theory, it is argued that if sociology is to understand the forces that transform the present status of society, it should pay more attention to the symbolic processes that occur in the literary experience, an activity that allows everyone to confront possible (fictional) situations and values and that, therefore, symbolizes a possible different social world.

Keywords: Literature, Fiction reading, Possible worlds, Sociology

\section{Introduction}

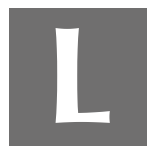

e propos de cet article est celui de discuter le statut contemporain de la sociologie de la littérature en tant que relation entre la littérature et le savoir sociologique. Poser la question en ces termes est prudent : il établit d'emblée une distance entre la littérature et le savoir sociologique manifestée par la présence de la préposition « et » qui sépare et relie les deux termes. Cette distance assumée ne préjuge donc pas du rapport existant entre la sociologie et la littérature, elle ouvre au contraire un espace de réflexion entre ces deux corpus discursifs. Et tant mieux.

J'y vois une marque de l'état de maturité de la réflexion dans les sciences sociales, qui a fait siennes les incertitudes qui pèsent sur la notion de savoir ou de connaissance dès lors qu'il s'agit du domaine des sciences humaines. L'impossibilité de séparer clairement le sujet et l'objet du savoir, consciente dès la naissance de la pensée dialectique, a rendu nécessaire la prudence qui transparaît dans ce titre. Elle a aussi produit une évolution remarquable en modifiant la place de la littérature dans cette dyade. Alors que l'expression 
« sociologie de la littérature » accordait à la littérature la place qu'occupe I'objet dans un processus cognitif à sens unique, on a pris conscience du fait que la frontière entre le discours sociologique et le discours littéraire était poreuse, qu'il y avait peut-être autant de sociologie dans la littérature que de littérature dans la sociologie.

Par ailleurs, c'est un des acquis de la phase structuraliste que d'avoir obligé toutes les disciplines à prendre conscience du fait qu'elles exercent leur volonté de savoir dans le champ contraignant du langage. La sociologie doit donc désormais elle aussi, tout comme l'histoire, non seulement s'interroger sur son lexique, où fourmillent les notions forgées au cours du temps sur des expériences sociales parfois vieilles de plusieurs siècles, mais aussi sur sa rhétorique, sa manière d'administrer la preuve, de construire la causalité sociale et enfin de s'adresser à son lecteur et de lui « représenter» le réel.

On parle souvent de la « sociologie de la littérature » comme s'il s'agissait d'une discipline dont l'objet et les méthodes étaient établies, dont il était possible par conséquent de restituer les moments significatifs de son élaboration. C'était, il faut bien l'avouer aujourd'hui, parler avec assurance de l'ombre d'un fantôme. L'excellent petit livre introductif de Paul Dirkx (2000), Sociologie de la littérature, paru plus récemment, éclaire ce caractère insaisissable de l'objet « sociologie de la littérature » en suggérant qu'il s'agit d'une « discipline en marche». Cela est sans doute vrai de toutes les branches des sciences humaines, mais on retiendra de cette formule habile qu'elle permet de mettre l'accent sur le fait que la constitution d'un objet susceptible de générer une discipline se fait par étapes, et que ce processus n'a peut-être pas de fin. Elle ressemble à une longue marche, scandée par des moments inégaux d'intensité intellectuelle et politique.

Depuis le début du XIXe siècle et les contrecoups de la Révolution Française (Cf. De Staël, 1999), bien des travaux ont contribué à construire ce champ disciplinaire. Ils ont été marqués d'abord, comme le rappelle Wolf Lepenies (1990), par la volonté de la sociologie naissante de délimiter son territoire en survalorisant sa différence d'avec la littérature. Il s'agissait d'établir une coupure nette entre le monde policé de la conversation dans 
lequel la pensée du XVIIle siècle s'était exprimée et le nouveau savoir sociologique. On reprochait en effet aux écrivains de ce temps de ne pas établir clairement de séparation entre le savoir commun et un discours « scientifique » construit, arborant les formes austères de la discipline académique. La science nouvelle exigeait un effort méthodique, qui devait être rendu sensible dans la forme même du discours. Dans certains cas, le jargon put apparaître comme la manifestation de la scientificité. Le dur exercice de la raison méthodique introduisit la raison mathématique dans les sciences humaines : celles-ci devaient reposer sur l'effacement de la singularité subjective au profit de la généralité statistique.

Aujourd'hui, la « sociologie de la littérature » n'est toujours pas une discipline, au sens où l'entend le monde académique. Il n'existe pas un corps de lois et de règles que tout chercheur puisse connaître et auxquelles il devrait se conformer. C'est une des raisons qui explique combien fragile est sa position dans l'espace institutionnel de l'enseignement supérieur. Tel le corps d'Osiris, son domaine est dispersé en une multiplicité de disciplines et de secteurs de connaissance qui vont de la sociolinguistique à l'histoire sociale du livre ou des écrivains, de l'analyse des genres littéraires à la critique sociale. Cet éparpillement explique à son tour la multiplicité des noms par lesquels les chercheurs désignent leur approche : « sociologie de la littérature », « sociologie littéraire », « sociologie du fait littéraire », «sociocritique » et bien d'autres.

\section{Difficulté de définir un objet un pour la sociologie de la littérature}

La sociologie académique évite en général d'affronter directement l'objet littéraire pour des raisons que Durkheim avait déjà énoncées dans son ouvrage séminal : Les Règles de la méthode sociologique. Afin de pouvoir traiter son objet en extériorité, comme il convient à toute science, Durkheim recommandait de veiller à définir l'objet du savoir par des « propriétés qui (lui) sont inhérentes ». C'est la condition pour éviter de s'en remettre à une 
« notion plus ou moins idéale » (Durkheim, 1947, p. 35). Or, comment définir la littérature par des propriétés qui lui sont inhérentes ? Existe-t-il, comme le suggérait Roman Jakobson en avançant la notion de «littérarité » (Literarnost), des signes susceptibles de définir la littérature à l'exclusion des autres types de textes ? Une telle tentative, qui a alimenté maintes réflexions dans les années 60-70, s'est soldée par un échec. L'horizon d'une telle science a reculé à mesure que la recherche avançait : elle abandonna. On peut d'ailleurs poser la même question au sujet de la fiction : possèdet-elle des propriétés qui lui sont inhérentes ?

Ne parvenant pas à définir la « littérarité » de la littérature, ou la « fictionnalité » de la fiction, la sociologie préfère aborder l'objet « littérature » de biais, par ses entours : la sociologie des publics, des auteurs, des critiques, de l'édition, de la lecture. C'est une stratégie qui s'en remet à une sorte de définition par prétérition de la littérature, fabriquant un objet qui, du moins, soit à la main du savoir sociologique. Dans la mesure où la littérature existerait en dehors de son noyau langagier et imaginaire, la sociologie disposerait d'un objet dont elle peut s'emparer. Il est toutefois évident que priver la définition de la littérature de ce qui fait son efficacité imaginaire, c'est se priver de la partie la plus essentielle de son appartenance au social, $c^{\prime}$ est renoncer à ce qui devrait au plus haut point intéresser une sociologie portant sur les dispositifs imaginaires à partir desquels se construisent les sociétés. Il convient donc de ne pas abandonner ces aspects, même si cette vision élargie ne suppose évidemment pas qu'on abandonne les approches du phénomène « littérature » par ce qui ne relève ni du texte ni des constructions imaginaires dont elle est l'occasion. C'est au contraire par la conjonction de ces différents plans que progresseront la recherche et la connaissance de l'existence sociale de la « littérature ».

\section{L'objet et la méthode}

Ce renoncement d'ordre méthodologique vient sans doute du fait qu'en ce qui concerne les objets symboliques, la sociologie se trouve, chez 
de nombreux sociologues, sous l'influence de la manière dont Durkheim posa la question du sacré. Pour ce dernier, le sacré s'élabore et s'objective dans le rituel et en découle. La réunion des croyants et les actes qu'ils produisent en se réunissant, - ferveur, amour, foi - construisent la société et la renforcent dans le même temps qu'ils construisent l'objet de la croyance : la religion. Sans le rituel, celle-ci n'existerait tout simplement pas. Appliquée au domaine de la littérature, cette approche survalorise la croyance en la valeur de l'œuvre d'art, laquelle se manifeste dans les rituels sociaux qui accompagnent la pratique de l'art ou de la littérature. Or, en insistant essentiellement, voire exclusivement, sur les aspects de rituel social qui entourent la fréquentation de l'art, parfois qualifiée $d^{\prime}$ " amour de l'art » ou $d^{\prime}$ " amour de la littérature », cette approche théorique a tendance à réduire la pratique sociale de la littérature à n'être que l'expression d'une concurrence entre capitaux symboliques, ayant pour conséquence, comme l'a fortement montré Pierre Bourdieu, de rendre dominants des comportements de distinction sociale.

La théorie de la distinction se double par ailleurs chez Bourdieu d'une théorie de l'usage « littéraire » du langage, tel que le pratiqueraient les écrivains. Ceux-ci feraient un usage esthétique du langage, s'adressant à leurs lecteurs sur le mode de la sensibilité. En privilégiant la sensibilité, I'écrivain permettrait à son lecteur de ne pas voir la vérité du réel, aveuglement qui ferait partie du plaisir littéraire. Bourdieu explique : «La mise en forme qu'il (l'écrivain) opère fonctionne comme un euphémisme généralisé et la réalité littérairement déréalisée et neutralisée qu'il propose lui permet de satisfaire (chez son lecteur) une volonté de savoir prête à se contenter de la sublimation que lui offre l'alchimie littéraire» (Bourdieu, 1992, p. 60).

À cet univers littéraire pensé sous l'angle des jeux de langage agissant sur la sensibilité, et qui détournerait la volonté de savoir de son objet, Bourdieu oppose la rigueur sociologique comme un ascétisme, un refus des fascinantes beautés de l'art. « La lecture sociologique rompt le charme. Mettant en suspens la complicité qui unit l'auteur et le lecteur dans le même rapport de dénégation de la réalité exprimée par le texte, elle révèle la vérité que le texte énonce, 
mais sur un mode tel qu'il ne la dit pas » (Bourdieu, 1992, p. 60-1). C'est toute la littérature qui se trouve ainsi privée de toute valeur cognitive. Dès lors, le sociologue ne saurait plus longtemps s'intéresser à l'œuvre littéraire, aussi bien pour des raisons méthodologiques que pour préserver l'efficacité de la recherche sociologique. Quels que soient les accords et les désaccords que l'on peut avoir avec les thèses de Bourdieu, la question fondamentale est que cette conception, toute platonicienne, de la littérature comme tromperie ne saurait être retenue. La littérature ne se situe pas sur le plan de l'énonciation de la vérité du réel. Elle n'est pas en concurrence sur ce plan avec la sociologie et celle-ci n'a pas lieu de prétendre mieux expliciter cette vérité du réel puisque là n'est pas l'objectif et la prétention de la littérature.

\section{Conjonctures intellectuelles}

Avant d'ouvrir un chemin empruntant une conception plus large de la littérature, il peut être intéressant de comprendre dans quel contexte celle que je viens de rappeler a pu être élaborée. Les théories sociologiques n'échappent pas plus que les autres aux mouvements idéologiques qui secouent les sociétés. Il est donc toujours utile de rapporter les unes aux autres, ne serait-ce que pour être capable de réfléchir sur la manière dont elles répondent aux conjonctures dans lesquelles elles apparaissent.

Arrêtons-nous d'abord à l'idée que la littérature peut entrer en concurrence avec le discours des sciences humaines. J'ai rappelé que la séparation de ces deux modalités discursives a été une des grandes affaires du XIXe siècle au moment de l'établissement de la sociologie comme discipline. Il faut ajouter à cela que ce débat a pris en France une importance majeure dans la mesure où la construction de l'identité nationale, notamment dans l'enseignement secondaire, s'est particulièrement appuyée sur le travail des écrivains. La littérature s'est trouvée investie d'une valeur d'exemplarité, dans l'ordre du maniement de la langue comme dans celui de la morale et de la politique. L'identité nationale de la France repose pour une part sur la référence, ritualisée et sacralisée, à son trésor littéraire. 
Enfin il faut replacer cette posture critique dans le moment intellectuel marqué par le structuralisme des années 60 et son attention portée sur les aspects métafictionnels de la littérature. Elle s'est trouvée alimentée par le sentiment d'enfin accéder à une science des textes, une révolution dans l'ordre de la littérature dont Tel Quel, après le Nouveau Roman, fut le porte-drapeau. Elle répondait également à la lassitude idéologique qui s'empara de l'intelligentsia après la fin de la guerre d'Algérie et l'entrée de la France dans la société de consommation. Plus rien de positif ne pouvait émaner des forces sociales et politiques, impasse que mit en forme la thèse de l'absolue domination des simulacres sur l'ensemble des actions sociales. Dans ce contexte, restaurer une distance proprement savante semblait offrir une alternative, alors même que toute praxis était mise en question.

Il s'agissait, en un mot, de rompre avec la tradition dont Jean-Paul Sartre avait été l'emblème, où toute réflexion valable était nécessairement articulée à des prises de positions politiques. Contre cette tradition alimentée encore par les derniers marxistes orthodoxes, le devoir critique consistait à arracher la pensée, et donc aussi la littérature, au devoir d'engagement. ${ }^{1}$ II fallait non seulement déconnecter le savoir des imprécisions inhérentes à l'usage du langage, ce que pointe la différence entre la rigueur philosophique du concept et les «charmes» de la narration et de la métaphore, mais également couper le savoir des affects et de l'univers trouble et complexe de la sensibilité individuelle.

Enfin, en comparant le pouvoir d'expliquer la réalité de la littérature et de la sociologie, la théorie de Bourdieu relance le débat sur la place de la fonction mimétique dans le processus littéraire. On se souvient de la fameuse

\footnotetext{
1 II faut noter que sur ce point, la notion sartrienne d'engagement diffère de ce qu'on pourrait appeler l'engagement sartrien. Dans Qu'est-ce que la littérature (1947), contrairement à ce qu'on lui fait souvent dire, Sartre n'exige pas de l'écrivain qu'il s'engage, sous-entendu qu'il prenne parti, mais Sartre lui fait comprendre qu'il est engagé, et qu'il ne saurait échapper à cette situation. Tout geste intellectuel est en même temps un geste politique, bien loin qu'il demande, dans la ligne du réalisme socialiste qu'il a toujours dénoncée, que l'écrivain ait un devoir moral d'écrire, par exemple, pour le peuple.
} 
métaphore imaginée par Stendhal à propos du roman : «Un roman, c>est un miroir que l’on promène le long d`un chemin. » Utilisée depuis fort longtemps, cette métaphore a causé de grands malentendus. Elle a servi, depuis Marx, à construire une théorie sociale de la littérature qui insistait d'abord sur le rapport mimétique du texte littéraire à la réalité sociale contemporaine de I'activité d'écriture. Cette tendance herméneutique fut souvent poussée jusqu'à la caricature et devint même, par un retournement moralisateur, une injonction adressée à l'écrivain de se tenir dans les limites d'un réalisme supposé, qualifié de « réalisme socialiste ». Or l'objet littéraire ne ressortit pas au monde des miroirs même si la mimesis lui est quasi consubstantielle (Cf. Auerbach, 1968). II ne saurait refléter la réalité, tout simplement parce que l'activité littéraire consiste à construire une réalité fictionnelle dans le langage. Si la littérature réfléchit à partir du monde réel, c'est peut-être à la manière des miroirs sans tain (two-way mirror). À la fois transparent et réfléchissant, le miroir sans tain tient un discours aux multiples facettes dans lequel la réalité se trouve mixée avec des mondes imaginaires qui appartiennent à l'écrivain, au lecteur, à la culture, au critique et au sociologue. Par conséquent, la question du sens de l'œuvre littéraire ne dépend pas seulement du « monde réel » auquel elle peut se référer, mais également de la confrontation de celui-ci avec d'autres à l'intérieur du texte littéraire.

Contre cette mauvaise compréhension du rôle de la mimesis dans le processus littéraire, et comme s'il fallait sauver l'activité de l'écrivain de toute dépendance au réel, certains théoriciens opposèrent l'idée de la « liberté » de l'écrivain. Insistant sur un prétendu jaillissement hors contraintes de la créativité artistique, ils voulaient occulter toute détermination de I'acte créatif. C'était évidemment construire une nouvelle illusion, dans la mesure où l'activité d'écriture travaille toujours à partir de « situations » qui constituent autant de contraintes, qu'elles soient langagières, sémiotiques, idéologiques ou matérielles. Le travail de l'écrivain dépend en effet non seulement d'un état historique et social de la langue, mais également de modèles littéraires qui sont une des matières symboliques auxquelles il ne 
peut échapper. Ces modèles lui servent dans la dynamique de l'échange qu'il instaure entre le monde fictionnel et des lecteurs qu'il interpelle. Au mieux, comme le soulignait Sartre, la liberté de l'écrivain consiste à prendre conscience des déterminations qui l'encadrent, et à faire œuvre (de liberté) à partir de celles-ci, contre et avec elles. C'est ce que visent à démontrer les grandes socio-biographies littéraires que Sartre a publiées sur Baudelaire (Sartre, 1963) et Flaubert (Sartre, 1971-1972). Or la définition de la littérature comme échange implique que l'on s'est affranchi de l'idée que l'œuvre littéraire est avant tout un texte.

La tradition occidentale a construit, au long des siècles, une conception de plus en plus rigoureuse de la notion de texte, à mesure que ses instruments d'analyse herméneutique se perfectionnaient eux-mêmes. On doit même dire, dans une perspective sociologique : à mesure qu'une catégorie de lecteurs, résidant souvent dans les couvents et les monastères, se faisaient les spécialistes de l'interprétation de texte. Cette professionnalisation de la lecture savante s'est faite d'abord à l'épreuve du texte sacré : la Bible. Les mouvements intellectuels qui, depuis la Renaissance, ont mis l'accent sur la référence stricte au texte de l'Ancien ou du Nouveau Testament ont eu pour effet paradoxal de faire éclater l'objet qu'ils prétendaient vénérer. Le scrupule quant à l'authenticité a conduit à repérer que les textes canoniques, qui étaient reçus comme sacrés, avaient en réalité été «composés», qu'ils constituaient des ensembles de fragments agencés au cours du temps par I'institution religieuse, ayant des origines locales, politiques et théologiques diverses. Dès lors le texte, qui à l'origine brillait dans l'unicité de la lumière divine dont il était censé être la révélation, se révèle un objet complexe aux origines plurielles, scintillant dans sa diversité sous le scalpel des herméneutes. Tel fragment a été rédigé par un groupe de docteurs de la Loi, tel autre par une secte dissidente de prédicateurs prophètes. Derrière la belle ordonnance que lui conférait l'espace du Livre, le texte laissait en son sein s'ouvrir des abîmes de complexité. Et pas seulement dans la Bible. 
Ce travail sur le texte sacré aura permis le développement des disciplines critiques et historiques appliquées aux textes. Ce ne sera que plus tard, avec ce qu'on a appelé the linguistic turn et la sémiotique structurale, que ces compétences philologiques et linguistiques seront mises au service de la lecture des textes profanes. Alors seulement une autre couche sociale, qui habitera désormais moins les couvents et les séminaires que les lycées et les universités, développera des compétences comparables à celles des anciens clercs. Ces travaux sur l'herméneutique, auxquels il faut ajouter l'évolution des mentalités qui les ont rendus possibles, conduiront, après un long cheminement qui passera par Bakhtine, à l'élaboration de la notion d'intertextualité et de métissage textuel. Celle-ci révélera dans la fiction littéraire des fonctionnements qui étaient efficaces depuis longtemps mais que la théorie n'apprenait à saisir que peu à peu. Laissant derrière elle les définitions de la littérature héritée des temps pré-littéraires - en gros avant le XVIIle siècle -, notre époque s'approche de ce qu'est devenue la pratique littéraire une fois émancipée des discours d'autorité : une plaque tournante, un point de rencontre entre imaginaires.

\section{La littérature et le savoir sociologique}

Il s'agit donc maintenant de réfléchir à la manière dont les sciences sociales de la littérature peuvent aborder l'objet « littérature » dans sa complexité à travers le kaléidoscope que constituent ses diverses dimensions : institutionnelles, fictionnelles et imaginaires. Les quelques réflexions qui viennent d'être proposées le définissent cet objet sur trois plans. La littérature ne se place pas sur l'isotopie de la vérité mimétique - et donc elle n'entre pas en concurrence avec la sociologie - dans la mesure où elle produit des dispositifs discursifs intégrant des mondes possibles; le texte littéraire entretient avec le monde réel un rapport mimétique nécessaire, lequel ne le décrit cependant pas de manière exhaustive dans la mesure où il est construit dans le langage et qu'il est donc fictionnel (plutôt que fictif). La littérature, enfin, et ce sera l'objet de notre dernière partie, est un échange 
qui implique ensemble un écrivain et un lecteur, au sein d'institutions sociales qui encadrent et nourrissent leurs relations. Cet échange prend place au cœur des processus symboliques qui constituent la société.

Depuis quelques décennies, le regard qui est posé sur la littérature a restauré la dimension pragmatique du phénomène littéraire. L'enjeu est précisément d'arracher l'univers fictionnel littéraire aux exigences « scientifiques » évoquées plus haut. L'absence de dénotation de la fiction (la fiction est nulle du point de vue dénotatif, elle ne reflète pas le réel !) n'a pas pour conséquence qu'elle ne produit pas de la connaissance. Mais pour résoudre l'impasse dans laquelle nous enferment ces théories, il faut changer le point de vue et adopter, comme la théorie de la fiction l'a finalement accepté, que les textes fictionnels existent « au-delà du vrai et du faux» comme dit joliment Gérard Genette. ${ }^{2}$ L'intérêt porté au texte littéraire par ses lecteurs implique nécessairement un degré d'immersion mimétique. Il suppose qu'on a cessé de croire que le but de la fiction serait de nous tromper. Au contraire, sa finalité littéraire, sa finalité sociale pourrait-on dire, est « d'élaborer des semblants ou des illusions ; les leurres qu'elle élabore sont simplement le vecteur grâce auquel elle peut atteindre sa finalité véritable, qui est de nous amener à nous engager dans une opération de modélisation, ou, pour le dire plus simplement, à nous amener à entrer dans la fiction » (Schaeffer, 1999, p. 199).

La modélisation fictionnelle ne se définit donc pas tant par des caractères propres, dont la linguistique et la sémiotique auraient le secret, elle n'est pas constituée par ses modalités esthétiques d'usage du langage, qui seraient en soi menteuses : la vérité référentielle n'est l'objet ni de l'art, ni de la littérature en général, ni de la fiction. Si la science cherche la vérité, elle se heurte, elle le sait bien, aux faiblesses du modèle langagier auquel elle a

${ }^{2}$ Le livre de Jean-Marie Schaeffer (1999) détaille de manière extrêmement claire et convaincante les impasses des théories sémantiques et les raisons de recourir à la perspective pragmatique introduite par Searle. Nous lui empruntons par la suite de nombreuses citations. La citation de Genette se trouve p. 210. On notera en outre l'importance, dans ce tournant pragmatique, du livre de John R. Searle (1982). 
recours, raison pour laquelle elle lui préfère la modélisation mathématique. Mais si la fiction ne trompe pas le lecteur, c'est qu'on n'est pas lecteur sans le savoir. Le caractère ambivalent de l'espace mental qu'ouvre la fiction littéraire provient du fait que celle-ci ne se sépare jamais totalement de la mimesis. Les mondes fictionnels ressemblent toujours suffisamment au monde « réel » pour permettre l'immersion dans un monde ressemblant, sur le mode du « comme si ». Ils s'en distinguent cependant, non moins nécessairement, en ce qu'ils ne peuvent jamais être qu'une modélisation de ces mondes, un choix signifiant effectué parmi des éléments qui sont partiellement repris de ces mondes réels. En même temps, le fait qu'ils sont marqués par un écart par rapport au réel constitue précisément ce qui garantit et éveille notre curiosité et notre intérêt. Il n'en va d'ailleurs pas autrement pour l'écrivain qui, même pour un « réaliste-naturaliste » comme Zola, après s'être amplement renseigné sur les moindres détails caractérisant la réalité qu'il va faire passer dans ses fictions, n'en fait une œuvre littéraire qu'à la faveur d'une invention qui doit tout et rien à cette réalité. Comme dit encore J.-M. Schaeffer : «l'immersion créatrice et I'immersion réceptrice ne sont que deux modalités différentes d'une même dynamique » (Schaeffer, 1999, p. 228).

La production littéraire de mondes fictionnels a donc pour but l'usage que le lecteur va en faire. Pour garantir cet échange, la stratégie de l'écrivain consiste à mettre en place tous les leurres qui, se fondant sur notre intérêt en général pour le monde dans lequel nous vivons, établiront les ponts qui nous aideront à nous y immerger. On les appellera avec Searle, « feintise » ou on parlera avec Ricœur, de «mondes du comme si », sachant que « la fonction de la feintise ludique est de créer un univers imaginaire et d'amener le récepteur à s'immerger dans cet univers, elle n'est pas de l'induire à croire que cet univers imaginaire est l'univers réel » (Schaeffer, 1999, p. 156).

Ecrivain et lecteur connaissent le contrat fictionnel qui les unit et qui régit un ordre de réalité différent de celui que suppose le savoir, tel que ses règles ont été posées dans le cadre de chacune de ses disciplines. Le contrat de lecture dans l'ordre de la fiction lève a priori la question du vrai 
et installe la relation par « delà le vrai et le faux». Ce contrat abolit, par la décision qu'il implique, toute pertinence à la question de la vérité ou de la fausseté. De fait la fiction, comme espace d'échange entre écrivain et lecteur, constitue une réalité sui generis. Et c'est cette réalité-là qui intéresse le sociologue. Elle est en effet un espace mental fictionnel autour duquel peuvent se cristalliser les différentes formes du monde changeant des représentations sociales. Si celui-ci était fixe, éternellement lié à l'état des choses telles qu'elles sont dans le monde habité par les acteurs, écrivain et lecteur, ce monde resterait immuable. Si la possibilité que le monde prenne d'autres formes n'apparaissait pas dans l'espace irréel des représentations, aucune force ne le pousserait à changer et il resterait immobile dans sa réalité présente, à peine touché par les lentes évolutions du système solaire. Mais le monde social dans lequel nous vivons ne cesse de se transformer au rythme où nous le transformons et où il nous transforme. L'espace fictionnel expérimental de ces transformations vacille entre réel et possible. Il est le monde en mutation constante que la fiction permet d'expérimenter.

Comment donc caractériser cette action de l'expérience fictionnelle dans le cadre plus large de l'expérience du réel ?

Le contrat de fiction, quelles qu'en soient les modalités, fusionnelles ou distancées, place le lecteur face à un monde possible. II contribue donc à produire ce que Goldmann (1964) appelait le champ de conscience possible, à savoir la marge de transformation de la conception qu'un lecteur se fait du monde, compte tenu de la place qu'il occupe dans le dispositif social global. Le possible est une des dimensions du réel, encadré par le lieu d'où chacun est capable de l'envisager. La notion de lieu ne signifie pas nécessairement, ici, une détermination spatiale, ni même temporelle. Le lieu peut consister en une position dans les rapports sociaux, constituer un lieu idéologique. Dans le rapport au possible qu'instaure le pacte fictionnel, se produit une expérimentation au cours de laquelle le lecteur traverse des situations, des rapports, des valeurs et des représentations. Dans la situation fictionnelle qu'offre la littérature, mais aussi les autres formes de fiction, le 
lecteur expérimente cette marge, il s'oblige à s'y confronter et à y mesurer ses propres valeurs, croyances et choix.

Par conséquent, la sociologie de la lecture fait partie intégrante des savoirs sociologiques sur la littérature. Dans les recherches que j'ai pu consacrer à cet aspect, il est clairement apparu que ce qui intéresse le lecteur, ce qui organise et justifie son activité de lecteur, c'est d'abord sa lecture elle-même, c'est elle qui est la causalité vibrante de son rapport au texte. Ce qui le motive, c'est l'investissement qu'il fera dans le jeu des actes et des valeurs possibles que le texte lui a rendus disponibles pour son expérimentation. «Autrement dit, ce qui est au centre de mon intérêt, c'est ce à quoi j'ai accès grâce à l'immersion mimétique, c'est-à-dire la modélisation fictionnelle d'un ensemble d'actions, d'événements, de sentiments etc. » (Schaeffer, 1999, p. 197).

La manipulation des univers imaginaires proposés par la littérature joue d'une manière tout à fait spécifique sur les contenus de valeur que véhiculent les œuvres. La socialisation par la manipulation des possibles implique une adhésion imaginaire, qui elle-même constitue la médiation à travers laquelle se produit une requalification de l'expérience, l'élévation de son niveau. Il y a une forme d'apprentissage qui passe par la possibilité de préfigurer un monde à travers l'expérimentation fictive. II faut en effet admettre que la littérature, telle que nous l'entendons dans I'occident démocratique, est un des lieux symboliques où, par excellence, s'expérimentent tous les types de rapports que nous entretenons avec nos semblables. Ils figurent et schématisent les rapports réels ou possibles des hommes et des femmes entre eux, ceux des citoyens avec l'environnement politique auquel ils appartiennent et d'une façon générale, l'ensemble des rapports d'altérité. Cette notion d'expérimentation s'entend aussi bien du travail de l'écrivain que de celui du lecteur. Pour ce dernier, la lecture est une mise en danger, il y affronte des mondes qu'il ne connaît ni ne maîtrise et dont, contrairement à l'écrivain, il ne peut changer le cours. Il prend des risques et ceux-ci participent pour une part à la manière qu'il aura de se vivre comme être social, plus tard, dans sa réalité quotidienne. 
Sans doute, avec l'évolution des techniques, cette fonction expérimentale qui a longtemps été l'apanage de la littérature, a-t-elle été relayée par d'autres univers imaginaires. On pense à ceux du cinéma, des nouveaux media et des jeux vidéo. Cependant, quelles que soient les différences, la structure fictionnelle de ces mondes imaginaires est organisée selon des schémas analogues à ceux de la littérature. Elle renvoie à des schèmes langagiers communs, même si la fragmentation de la réception propre aux modalités les plus récentes de fiction, pourrait changer la donne et mettre en danger les valeurs d'exemplarité dont l'expérience de la littérature fait commerce.

En tant qu'elle imagine et formalise des mondes, ou des schèmes de mondes et de relations, la fiction opère, comme on l'a vu par une «modélisation mimétique ». Ce n'est pas le lieu de développer la question des modalités psychologiques ou mentales mises en jeu dans cette activité d'autostimulation imaginative qu'est la lecture. Ce qui devrait en revanche intéresser la sociologie, c'est de voir comment les schématisations qui sont à l'œuvre dans ces processus ont elles-mêmes des effets sur les prises de position des acteurs dans la société et comment elles se répartissent de manière spécifique et différenciée selon les groupes sociaux auxquels appartiennent les écrivains comme les lecteurs. Aborder de front, et de manière expérimentales, l'énergie propre de ces schématisations ouvrirait la voie à une sociologie des transformations sociales. On échapperait ainsi, en se mettant à l'écoute des mondes qui s'esquissent dans l'imaginaire à la faveur de l'échange fictionnel, au caractère désespérément rétrospectif de l'analyse sociologique. En donnant toute sa place à la pratique de la littérature au cœur de l'imagination sociale, la sociologie se donnerait quelques instruments qui lui permettraient peut-être de mieux appréhender la dimension dynamique du mouvement des formes sociales. 
Jacques Leenhardt est Directeur d'Études à l'École des Hautes Études en Sciences Sociales (EHESS), Paris, où il dirige l'équipe de recherche EFISAL : Fonction imaginaires et sociales des arts et des littératures.

ఏj jacques.leenhardt@ehess.fr

\section{Références bibliographiques}

1. AUERBACH, Erich. Mimesis : La représentation de la réalité dans la littérature occidentale. Paris : Gallimard, 1968.

2. BOURDIEU, Pierre. Les Règles de l'art. Paris : Seuil, 1992.

3. DE STAËL, Madame. De la Littérature considérée dans ses rapports avec les Institutions Sociales (1798-1800). Paris : GF Flammarion, 1999.

4. DIRKX, Paul. Sociologie de la littérature. Paris : Armand Colin, 2000.

5. DURKHEIM, Émile. Les Règles de la méthode sociologique. Paris : Presses universitaires de France, 1947.

6. LEENHARDT, Jacques; JOZSA, Pierre. Lire la lecture. Essai de sociologie de la lecture. Paris : I'Harmattan, 1999.

7. LEPENIES, Wolf. Les trois cultures, entre science et littérature, avènement de la sociologie. Paris : Maison des Sciences de l'Homme, 1990.

8. SARTRE, Jean-Paul. Baudelaire. Paris : Idées-NRF, 1963.

9. SARTRE, Jean-Paul. L'Idiot de la famille : Gustave Flaubert de 1821 à 1857, Trois tomes. Paris : Gallimard, 1971-1972.

10. SCHAEFFER, Jean-Marie. Pourquoi la fiction ? Paris : Seuil, 1999.

11. SEARLE, John R. Sens et expression, Etudes de théorie des actes de langage. Paris : Minuit, 1982. 